\title{
Magnetic anisotropy in ferromagnetic Josephson junctions
}

M. Weides

Citation: Appl. Phys. Lett. 93, 052502 (2008);

View online: https://doi.org/10.1063/1.2967873

View Table of Contents: http://aip.scitation.org/toc/apl/93/5

Published by the American Institute of Physics

\section{Articles you may be interested in}

Switching at small magnetic fields in Josephson junctions fabricated with ferromagnetic barrier layers Applied Physics Letters 104, 022602 (2014); 10.1063/1.4862195

Spin-transfer switching of orthogonal spin-valve devices at cryogenic temperatures Journal of Applied Physics 115, 17C725 (2014); 10.1063/1.4865464

Spin-polarized supercurrents for spintronics

Physics Today 64, 43 (2011); 10.1063/1.3541944

Controllable Josephson current through a pseudospin-valve structure Applied Physics Letters 84, 1153 (2004); 10.1063/1.1646217

High quality ferromagnetic 0 and $\pi$ Josephson tunnel junctions Applied Physics Letters 89, 122511 (2006); 10.1063/1.2356104

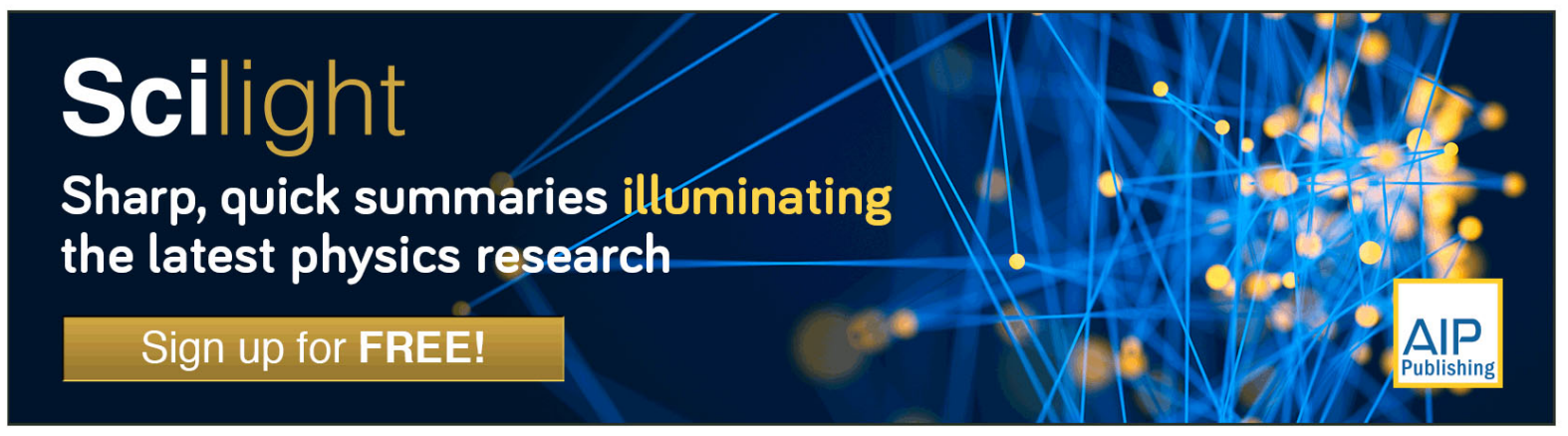




\title{
Magnetic anisotropy in ferromagnetic Josephson junctions
}

\author{
M. Weides \\ Center of Nanoelectronic Systems for Information Technology and Institute of Solid State Research, \\ Research Centre Jülich, D-52425 Jülich, Germany
}

(Received 26 May 2008; accepted 13 July 2008; published online 5 August 2008)

\begin{abstract}
Magnetotransport measurements were done on $\mathrm{Nb} / \mathrm{Al}_{2} \mathrm{O}_{3} / \mathrm{Cu} / \mathrm{Ni} / \mathrm{Nb}$ superconductorinsulator-ferromagnet-superconductor Josephson tunnel junctions. Depending on ferromagnetic Ni interlayer thickness and geometry, the standard $(1 d)$ magnetic field dependence of critical current deviates from the textbook model for Josephson junctions. The results are qualitatively explained by a short Josephson junction model based on anisotropy and $2 d$ remanent magnetization. (C) 2008 American Institute of Physics. [DOI: 10.1063/1.2967873]
\end{abstract}

Superconductivity $(\mathrm{S})$ and ferromagnetism $(\mathrm{F})$ in thin layered films have now been studied during some decades. ${ }^{1}$ In SF bilayers the superconductivity may be nonuniform, i.e., the Cooper pair wave function extends to the ferromagnet with an oscillatory behavior. In Josephson junctions (JJs) based on $s$-wave superconductors the phase coupling between the superconducting electrodes can be shifted by $\pi$ when using a ferromagnetic barrier with an appropriate chosen thickness $d_{F}$, i.e., SFS or SIFS-type junctions (I: insulating tunnel barrier). Only in recent years the experimental realization of $\pi$ JJs was successful. In particular, the $\pi$ coupling was demonstrated by varying the temperature, ${ }^{2-4}$ the thickness of the F-layer, ${ }^{4-7}$ or measuring the current-phase relation of JJs incorporated into a superconducting loop. ${ }^{8-10}$ The coupling can also change within a single JJ by a steplike F-layer, i.e., one-half is a $0 \mathrm{JJ}$ and the other half is a $\pi \mathrm{JJ} .{ }^{11,12}$

For useful classical or quantum circuits based on SFS/ SIFS JJs, a large critical current density $j_{c}$ (small Josephson penetration depth $\lambda_{J}$ ) and a high $I_{c} R$ product are needed. ${ }^{13,14}$ Up to now the limiting factor is the low $j_{c}$ due to a strong Cooper pair breaking inside F-layer. Alloys of magnetic and nonmagnetic atoms such as $\mathrm{NiCu}$ face problems of clustering $^{15}$ and magnetic scattering. ${ }^{4,7}$ Promising experiments using strong magnets ${ }^{5,16-19}$ were published.

Shape anisotropy of magnetic interlayer may provoke a not flux-closed domain structure and consequently a shift of critical current diffraction pattern $I_{c}(H){ }^{18}$ In experiments, ${ }^{2,4,6,18,19}$ the SFS/SIFS JJs had nearly mirror symmetrical $I_{c}(H)$, i.e., the effective shift along the $H$-axis is small, usually less than one flux quantum $\Phi_{0}$. This is explained by a multidomain state of F-layer with a very small net magnetization. However, up to now the $2 d$ nature of thin-film magnetism was disregarded.

In this letter the $I_{c}(H)$ dependence for remanent $2 d$ magnetization of F-layer is systematically studied. First, the maximal flux from F-layer is estimated. Second, the $I_{c}(H)$ pattern considering $2 d$ in-plane magnetization is calculated for different aspect ratios. Third, the $I_{c}(H)$ pattern is measured along both magnetic axes for various junction geometries and $d_{F}$.

The maximal shift of $I_{c}\left(H_{y}\right)$ is estimated for a strong magnet, i.e., $\mathrm{Ni}$, being magnetized fully in plane and along the $y$-axis. The atomic magnetic momentum is $0.6 \mu_{\mathrm{B}}{ }^{20}$ the specific density $\rho$ is $8.9 \mathrm{~g} / \mathrm{cm}^{3}$ (bulk), and magnetization $\mu_{0} M=0.64 \mathrm{~T}$. A cross section of length $L_{x}=100 \mu \mathrm{m}$ and F-layer thickness $d_{F}=3 \mathrm{~nm}$ encloses a magnetic flux $\Phi_{M}$
$=d_{F} L_{x} \mu_{0} M$. The total magnetic flux $\Phi$ through the $\mathrm{JJ}$ is the applied field flux $\Phi_{H}=\left(2 \lambda_{L}+d_{F}\right) L_{x} H_{y}$ (London penetration depth $\lambda_{L}=90 \mathrm{~nm}$ ) plus $\Phi_{M}$, i.e., $\Phi=\Phi_{H} \pm \Phi_{M}$ $=8.85 \Phi_{0} H_{y} / \mathrm{mT} \pm 92 \Phi_{0}$. The $I_{c}\left(H_{y}\right)$ pattern is shifted by 92 periods from the center. This simple calculation neglects dead magnetic layer, ${ }^{21}$ as found in SFS/SIFS JJs, ${ }^{4,7,19}$ and demagnetizing by domains. Real Ni films tend to form complex magnetization profiles (in/out of plane) and domain structures as function of $d_{F}{ }^{22}$ Integral magnetization measurements in SF layers show a complex behavior as a function of temperature, applied field, and sample history, ${ }^{23,24}$ e.g., SF structures spontaneously alter their stray field by changing magnetic domain distribution. ${ }^{25}$ The local magnetization depends on stray fields from neighbor domains, flux focusing from S-electrodes, ${ }^{18,26}$ or on bias induced spin accumulation at $\mathrm{F} / \mathrm{S}$ interface. ${ }^{27}$ It is generally agreed that the average magnetization in SFS/SIFS JJs is much smaller than the maximum magnetization estimated above. However, as shown below even a remanent $2 d$ magnetization of $1 \%$ of the maximal value may notably change the $I_{c}(H)$ pattern.

A qualitative model for $I_{c}(H)$ in presence of a uniform fixed $2 d$ magnetization $\vec{M}$ is derived. The short JJ model

$$
I_{c}(H)=I_{c}^{0}\left|\frac{\sin \left(\pi \frac{\Phi}{\Phi_{0}}\right)}{\pi \frac{\Phi}{\Phi_{0}}}\right| \text { and } \Phi=\Phi_{H} \pm \Phi_{M}
$$

is modified by $2 d$ distributions of applied field flux $\Phi_{H}$ and magnetization $\Phi_{M}$ with

$$
\Phi=\left|\left[\left(2 \lambda_{L}+d_{F}\right)\left(\begin{array}{cc}
0 & H_{x} \\
H_{y} & 0
\end{array}\right) \pm\left(\begin{array}{cc}
\alpha & 0 \\
0 & \alpha
\end{array}\right)\right] \times\left(\begin{array}{c}
L_{x} \\
L_{y}
\end{array}\right)\right| .
$$

$\vec{M}=\alpha\left(L_{x}, L_{y}\right)$ is assumed to be orientated in-plane (Meissnerscreening of S-electrodes) along the diagonal (i.e., longest) axis of sample (rough approximation for magnetic shape anisotropy). The magnetization $\vec{M}$ is several orders of magnitude smaller than the upper limit given by a fully saturated magnetic layer. Note that the easy axis of ferromagnetic film can be determined by the magnetic field during deposition, too. The model for $\Phi_{M}$ is just exemplary for the effect of $2 d$ in-plane magnetization in $I_{c}(H)$.

In Fig. 1, the surface plot of $I_{c}\left(H_{x}, H_{y}\right)$ is depicted for various $\alpha$ and geometries. The position of $I_{c}^{0}$ is shifted from the center $\left(H_{x}=H_{y}=0 \mathrm{mT}\right)$ in opposite direction of $\vec{M}$. Figure 

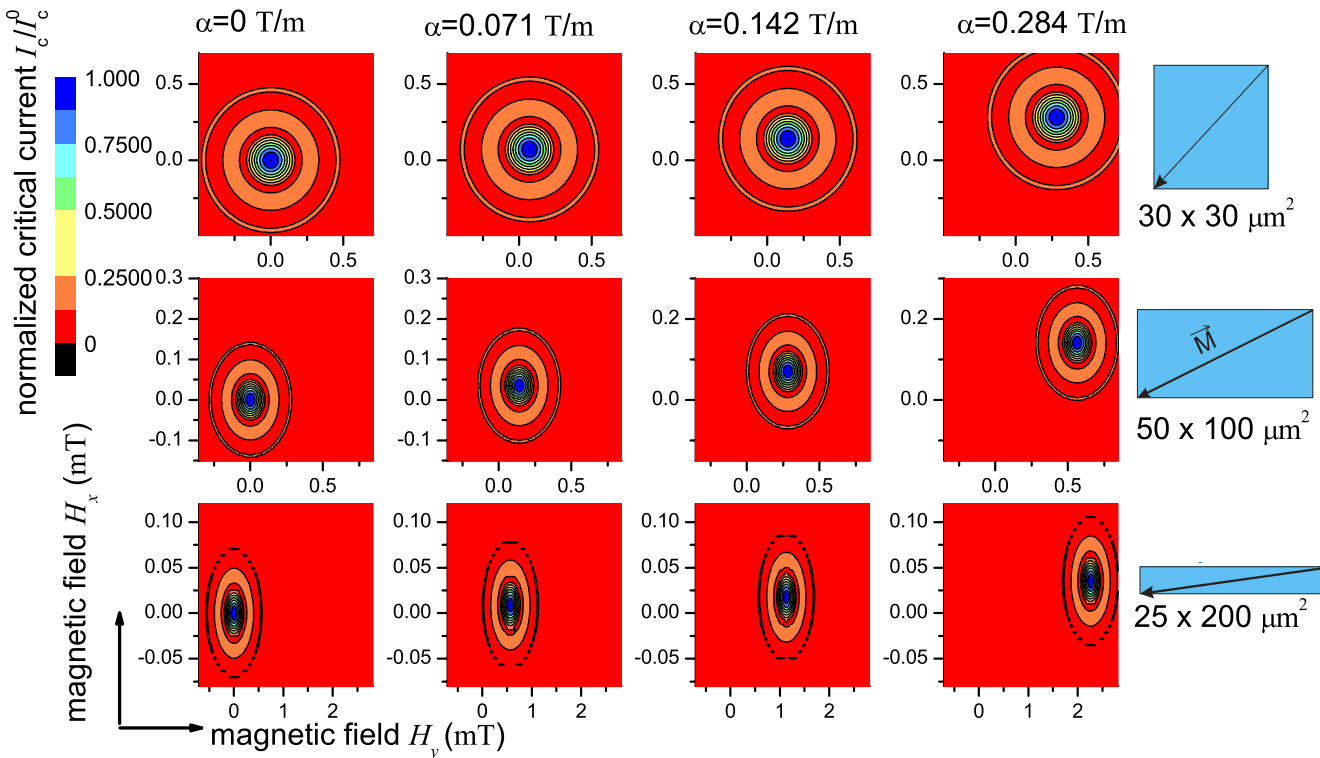

FIG. 1. (Color online) Calculated surface plot of $I_{c}\left(H_{x}, H_{y}\right)$ for different geometries and remanent magnetizations $\alpha$. The magnetization vector $\vec{M}$ points from top right to bottom left corner (arrow) and $I_{c}^{0}$ is shifted in opposite direction.

2 depicts $I_{c}\left(H_{x}, 0\right)$ and $I_{c}\left(0, H_{y}\right)$ patterns. These graphs resemble standard $1 d I_{c}(H)\left(H=H_{x}, H_{y}\right)$ measurements. For some $|\vec{M}| \neq 0 \mathrm{~T}$, a single-peaked $I_{c}\left(H_{x}\right), I_{c}\left(H_{y}\right)$ pattern is calculated, which—on first glance-resembles a shifted $|\sin (H) / H|$ Fraunhofer pattern. However, the maximum $I_{c}$ is smaller than the real $I_{c}^{0}$ and the height of side maxima does not obey the expected value. For example, the $I_{c}\left(H_{x}, 0\right)$ pattern of $30 \times 30 \mu \mathrm{m}^{2}$ sample and $\alpha=0.071 \mathrm{~T} / \mathrm{m}$ is shifted by less than $\Phi_{0}$ and its maximum $I_{c}$ is already reduced to $\sim 0.8 I_{c}^{0}$. This simple $I_{c}\left(H_{x}, H_{y}\right)$ model may qualitatively explain some experimental observations (Fig. 3) on SIFS- type JJs with $\mathrm{Ni}$ interlayer.

For experiment JJs with similar areas, i.e., $30 \times 30,50$ $\times 100$, and $25 \times 200 \mu \mathrm{m}^{2}$, were fabricated. The SIFS multilayer was sputter deposited with $d_{F}$ ranging from 1 to $6 \mathrm{~nm}$. The tunnel barrier was formed for $30 \mathrm{~min}$ at a partial oxygen pressure of 0.1 mbar. After oxidation a $2 \mathrm{~nm}$

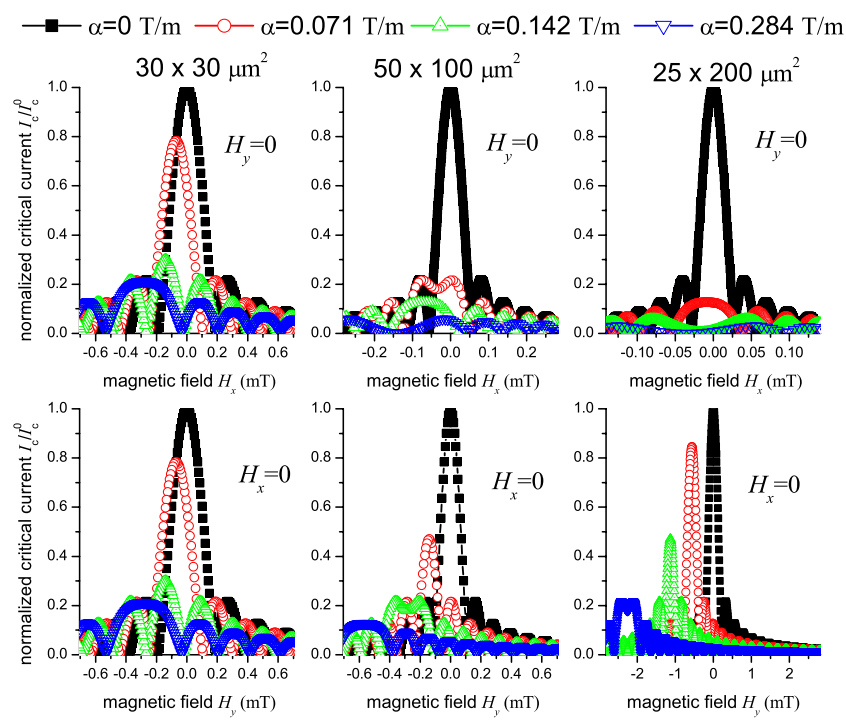

FIG. 2. (Color online) Calculated $I_{c}$ as function of $1 d$ magnetic field, $I_{c}\left(H_{x}, 0\right)$ (top), and $I_{c}\left(0, H_{y}\right)$ (bottom) for the three geometries and various $\alpha$. A substantial deviation from ideal $(\alpha=0 \mathrm{~T} / \mathrm{m})$ pattern appears for already small magnetization vector $\vec{M}=\alpha\left(L_{x}, L_{y}\right)$.
$\mathrm{Cu}$ film was inserted. All JJs were deposited in a single run by shifting the substrate and target to obtain a wedge-shaped $\mathrm{Ni}$ layer. ${ }^{28}$ Normal state and subgap resistance indicate a small junction to junction variation. The $I V$ and $I_{c}\left(H_{x}\right)$, $I_{c}\left(H_{y}\right)$ characteristics were measured at $4.2 \mathrm{~K}$ for two sets of samples $\left(d_{F}=2.2\right.$ and $\left.3.9 \mathrm{~nm}\right)$. Cooldown was done in zero field and thermal cycling up to $\approx 15$ and $300 \mathrm{~K}$ to check reproducibility. Measurements were made in a liquid He dip probe using low-noise homemade electronics and roomtemperature voltage amplifier. The fields $\left(H_{x}, 0\right),\left(0, H_{y}\right)$ were applied in plane of the sample and parallel the junctions axis (Fig. 1). The voltage criteria $V_{c}$ for $I_{c}(H)$ determination were $0.2-1 \mu \mathrm{V}$. A lower subgap resistance for $d_{F}=3.9 \mathrm{~nm}$ sample leads to larger offset currents. Positive and negative current branches of IVC had similar magnetic field dependence $+I_{c}\left(H_{y}\right) \simeq\left|-I_{c}\left(H_{y}\right)\right|$. Magnetic field was swept between $\pm 1.5 \mathrm{mT}$ for all samples. All junctions had their lateral sizes
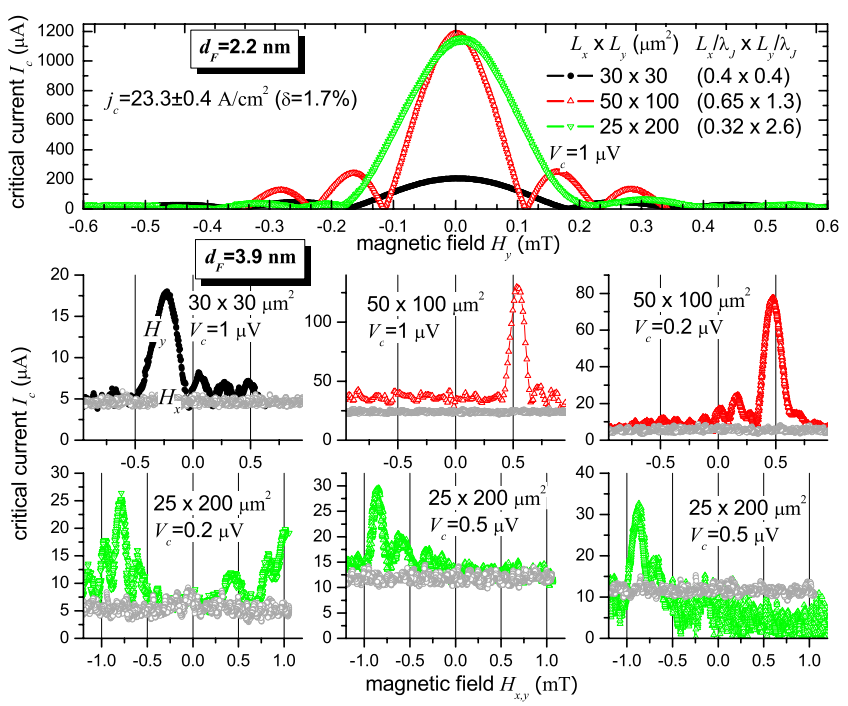

FIG. 3. (Color online) Measured $I_{c}\left(H_{y}\right)$ pattern of SIFS JJs for thin (top) and thick (bottom) Ni layers and different geometries. $I_{c}\left(H_{x}\right)$ pattern of thick $\mathrm{Ni}$ layer is plotted in gray. Onset of magnetic anisotropy effects in Ni layer is between 2.2 and $3.9 \mathrm{~nm}$. 
comparable or smaller than the Josephson penetration length $\lambda_{J}$, except the longest sample $\left(d_{F}=2.2 \mathrm{~nm}, 25 \times 200 \mu \mathrm{m}^{2}\right)$, whose $L_{y}$ is not strictly inside the short JJ limit.

The $d_{F}=2.2 \mathrm{~nm}$ samples showed very regular $I_{c}\left(H_{y}\right)$ pattern. All maximum $I_{c}$ 's were nearly centered and the spread of $j_{c}$ was $\sim 1.7 \%$, as determined from the maximum $I_{c}$ 's. The $I_{c}\left(H_{x}\right)$ pattern is symmetric, too (not shown). The oscillation period of $I_{c}\left(H_{y}\right)$ was determined by magnetic cross section $\sim 1 / L_{x}$, and nearly independent of aspect ratio. No indication for a distorted supercurrent transport due to alloying at the $\mathrm{Nb} / \mathrm{Ni}$ interface ${ }^{29}$ can be found. Effects due to magnetic anisotropy were not detectable, either because the samples were still inside the dead magnetic layer or the anisotropy was absent or totally out of plane.

The $d_{F}=3.9 \mathrm{~nm}$ samples had completely different $I_{c}\left(H_{y}\right)$ pattern showing in-plane anisotropy with some characteristic features. All maximum $I_{c}$ 's were shifted from the center, and the amplitude of shift increased with $L_{y}$, i.e., $\approx 0.24 \mathrm{mT}$ for $30 \times 30 \mu \mathrm{m}^{2}, \approx 0.5 \mathrm{mT}$ for $50 \times 100 \mu \mathrm{m}^{2}$, and $\approx 0.8 \mathrm{mT}$ for $25 \times 200 \mu \mathrm{m}^{2}$ samples. The direction of shift varies between samples even if they were cooled and measured at the same time (random polarity of magnetic configuration). The position of main peak of $I_{c}\left(H_{y}\right)$ was reproducible after thermal cycling to $300 \mathrm{~K}$. The width of main maxima (measured at offset line) was not strictly $\sim 1 / L_{x}$, and varies from sample to sample. The pattern were asymmetric, i.e., the height of same-order side maxima differed, probably due to nonuniform flux guidance in F-layer and reorientation of domains.

By rotating the magnetic field by $90^{\circ}$, i.e., measuring in $I_{c}\left(H_{x}\right)$ mode, low $I_{c}$ 's, being nearly independent of $H_{x}$, were detected. Even the squared shaped $30 \times 30 \mu \mathrm{m}^{2} \mathrm{JJ}$ had an almost flat $I_{c}\left(H_{x}\right)$ pattern. This indicates some magnetic crystallographic anisotropy along the $y$-axis, probably caused by magnetron sputter deposition. Small deviations of $I_{c}(0,0)$ for $I_{c}\left(H_{x}, 0\right)$ and $I_{c}\left(0, H_{y}\right)$ measurements can be related to variations of magnetic configuration by the unshielded sample handling at $300 \mathrm{~K}$. A considerable spread of maximum $I_{c}$ (Fig. 3) can be already seen for JJs with the same geometry, which is even increased by considering the maximum $j_{c}$ for different geometries. Simulations (Fig. 2) show that already a moderate magnetization $\vec{M}(\alpha<0.1 \mathrm{~T} / \mathrm{m})$ yields very different maximum $I_{c}$ 's of $I_{c}\left(H_{x}\right)$ and $I_{c}\left(H_{y}\right)$. A sample to sample variation of direction and amplitude of $\vec{M}$ explains the data spread of Fig. 3. Obviously, this leads to very large variations in the $I_{c}\left(d_{F}\right)$ dependence.

In literature, the $I_{c}(H)$ pattern of SFS/SIFS JJs with comparable strong magnets were either shown for samples with thin $d_{F}$ (Ref. 18) or had deviations from the ideal $|\sin (H) / H|$ form. For example, the maximum $I_{c}$ in Ref. 19 [Fig. 5 (inset)] is too small compared to the first side maxima. These samples were small in area $\left(\leqslant 1 \mu \mathrm{m}^{2}\right)$, and the F-layer could have been in single domain state. As $I_{c}$ varied smoothly with $H$, either $\vec{M}$ rotated softly, or a multidomain structure with averaged $2 d$ magnetization existed. For both cases the $2 d$ nature of remanent magnetization may have suppressed the maximum $I_{c}$ determined from $I_{c}(H)$ pattern-below the real $I_{c}^{0}$.

In summary, the $I_{c}(H)$ pattern along both field axes of SIFS JJs with Ni interlayer were measured. Assuming magnetic anisotropy the characteristic features, i.e., shift or absence of central peak, can be qualitatively reproduced by simulations. As conclusion, the $1 d I_{c}(H)$ pattern in the presence of magnetic anisotropy cannot yield the real $I_{c}^{0}$. Future experiments on SFS/SIFS JJs should be done by two-axis $I_{c}\left(H_{x}, H_{y}\right)$ scan of JJs with well-controlled magnetic interlayer domain configuration. Superconducting spintronic devices such as FSF spin valves and $\pi, 0-\pi$ JJs are promising candidates for future classical and quantum computers. As shown by this letter, control of magnetic anisotropy and magnetic domain configuration is essential for phaseswitchable FSF/SFIFS or SFS/SIFS devices.

M.W. thanks D. Sprungmann and A. Bannykh for assistance, and DFG for support (Project No. WE 4359/1-1).

${ }^{1}$ A. I. Buzdin, Rev. Mod. Phys. 77, 935 (2005).

${ }^{2}$ V. V. Ryazanov, V. A. Oboznov, A. Y. Rusanov, A. V. Veretennikov, A. A. Golubov, and J. Aarts, Phys. Rev. Lett. 86, 2427 (2001).

${ }^{3}$ H. Sellier, C. Baraduc, F. Lefloch, and R. Calemczuk, Phys. Rev. B 68 , 054531 (2003).

${ }^{4}$ M. Weides, M. Kemmler, E. Goldobin, D. Koelle, R. Kleiner, H. Kohlstedt, and A. Buzdin, Appl. Phys. Lett. 89, 122511 (2006).

${ }^{5}$ Y. Blum, A. Tsukernik, M. Karpovski, and A. Palevski, Phys. Rev. Lett. 89, 187004 (2002).

${ }^{6}$ T. Kontos, M. Aprili, J. Lesueur, and X. Grison, Phys. Rev. Lett. 89, 137007 (2002).

${ }^{7}$ V. A. Oboznov, V. V. Bol'ginov, A. K. Feofanov, V. V. Ryazanov, and A. I. Buzdin, Phys. Rev. Lett. 96, 197003 (2006).

${ }^{8}$ A. Bauer, J. Bentner, M. Aprili, M. L. D. Rocca, M. Reinwald, W. Wegscheider, and C. Strunk, Phys. Rev. Lett. 92, 217001 (2004).

${ }^{9}$ V. V. Ryazanov, V. A. Oboznov, A. V. Veretennikov, and A. Y. Rusanov, Phys. Rev. B 65, 020501 (2001).

${ }^{10}$ W. Guichard, M. Aprili, O. Bourgeois, T. Kontos, J. Lesueur, and P. Gandit, Phys. Rev. Lett. 90, 167001 (2003).

${ }^{11}$ M. Weides, M. Kemmler, H. Kohlstedt, R. Waser, D. Koelle, R. Kleiner, and E. Goldobin, Phys. Rev. Lett. 97, 247001 (2006).

${ }^{12}$ M. Weides, C. Schindler, and H. Kohlstedt, J. Appl. Phys. 101, 063902 (2007)

${ }^{13}$ A. V. Ustinov and V. K. Kaplunenko, J. Appl. Phys. 94, 5405 (2003).

${ }^{14}$ T. Kato, A. A. Golubov, and Y. Nakamura, Phys. Rev. B 76, 172502 (2007).

${ }^{15}$ R. W. Houghton, M. P. Sarachik, and J. S. Kouvel, Phys. Rev. Lett. 25, 238 (1970).

${ }^{16}$ V. Shelukhin, A. Tsukernik, M. Karpovski, Y. Blum, K. B. Efetov, A. F. Volkov, T. Champel, M. Eschrig, T. Löfwander, G. Schön, and A. Palevski, Phys. Rev. B 73, 174506 (2006).

${ }^{17} \mathrm{~J}$. W. A. Robinson, S. Piano, G. Burnell, C. Bell, and M. G. Blamire, Phys. Rev. Lett. 97, 177003 (2006).

${ }^{18}$ C. Bell, R. Loloee, G. Burnell, and M. G. Blamire, Phys. Rev. B 71 180501 (2005).

${ }^{19}$ J. W. A. Robinson, S. Piano, G. Burnell, C. Bell, and M. G. Blamire, Phys Rev. B 76, 094522 (2007).

${ }^{20}$ S. A. Ahern, M. J. C. Martin, and W. Sucksmith, Proc. R. Soc. London 248, 145 (1958).

${ }^{21}$ L. Liebermann, J. Clinton, D. M. Edwards, and J. Mathon, Phys. Rev. Lett. 25, 232 (1970)

${ }^{22}$ G. Gubbiotti, G. Carlotti, M. G. Pini, P. Politi, A. Rettori, P. Vavassori, M. Ciria, and R. C. O'Handley, Phys. Rev. B 65, 214420 (2002).

${ }^{23}$ C. Monton, F. de la Cruz, and J. Guimpel, Phys. Rev. B 77, 104521 (2008)

${ }^{24}$ A. G. Joshi, S. A. Kryukov, L. E. D. Long, E. M. Gonzalez, E. Navarro, J. E. Villegas, and J. L. Vicent, J. Appl. Phys. 101, 09G117 (2007).

${ }^{25}$ S. V. Dubonos, A. K. Geim, K. S. Novoselov, and I. V. Grigorieva, Phys. Rev. B 65, 220513 (2002).

${ }^{26}$ H. Wu, J. Ni, J. Cai, Z. Cheng, and Y. Sun, Phys. Rev. B 76, 024416 (2007).

${ }^{27}$ F. J. Jedema, B. J. van Wees, B. H. Hoving, A. T. Filip, and T. M. Klapwijk, Phys. Rev. B 60, 16549 (1999).

${ }^{28}$ M. Weides, K. Tillmann, and H. Kohlstedt, Physica C 437-438, 349 (2006).

${ }^{29}$ H. Chen, Y. Du, H. Xu, Y. Liu, and J. C. Schuster, J. Mater. Sci. 40, 6019 (2005). 